Y.-H. Chu, N.B. Suntzeff, J.E. Hesser, and D.A. Bohlender, eds.

\title{
The Kinematic Structure of the Supergiant Shell LMC 2
}

\author{
Sean Points, You-Hua Chu, Sungeun Kim, Robert Gruendl \\ Astronomy Dept., Univ. of Illinois, Urbana, IL 61801, USA
}

R. Chris Smith

Dept. of Astronomy, Univ. of Michigan, Ann Arbor, MI 48109, USA

Steve Snowden

NASA/Goddard Space Flight Center, Greenbelt, MD 20771, USA

Wolfgang Brandner

JPL/IPAC, Pasadena, CA 91125, USA

\section{Introduction}

LMC 2 was one of the first supergiant shells in the Large Magellanic Cloud (LMC) to be identified, based on the detection of long, curved $\mathrm{H} \alpha$ filaments extending over 900 pc (Goudis \& Meaburn 1978). LMC 2 is located to the east of the active star formation region south of 30 Doradus. LMC 2 is a spectacular supergiant shell in the LMC, having the most coherent filamentary structure and the highest X-ray surface brightness. As shown in Fig. 1, the diffuse X-ray emission from LMC 2 is bounded by the $\mathrm{H} \alpha$ filaments in the north and east. A bright X-ray arc is seen in the southwest quadrant, extending from N 158 and N 159.

The kinematic structure of LMC 2 has been investigated by various groups with conflicting results. Caulet et al. (1982) studied the kinematics of the $10^{4} \mathrm{~K}$ ionized gas and concluded that LMC 2 was a coherently expanding shell-like structure. Meaburn et al. (1987) obtained profiles of the $\mathrm{H}_{\mathrm{I}} 21 \mathrm{-cm}$ emission line toward LMC 2. They detected no evidence that LMC 2 was coherently expanding. We have obtained high-resolution echelle spectra of the $\mathrm{H} \alpha$ line and Austrailia Telescope Compact Array (ATCA) aperture synthesis maps of the H I 21-cm line emission of LMC2 to study its dynamics.

\section{Kinematic Structure of LMC 2}

Our echelle spectra along three cuts toward LMC 2 (designated EI, EII, \& E III in Fig. 1) reveal two major velocity components of the ionized gas at $\mathrm{V}_{\text {hel }} \sim 250$ and $275 \mathrm{~km} \mathrm{~s}^{-1}$. The velocity structure of these components across the echelle cuts does not show a velocity variation conforming to that expected in an expanding hemisphere (Points et al. 1999). 


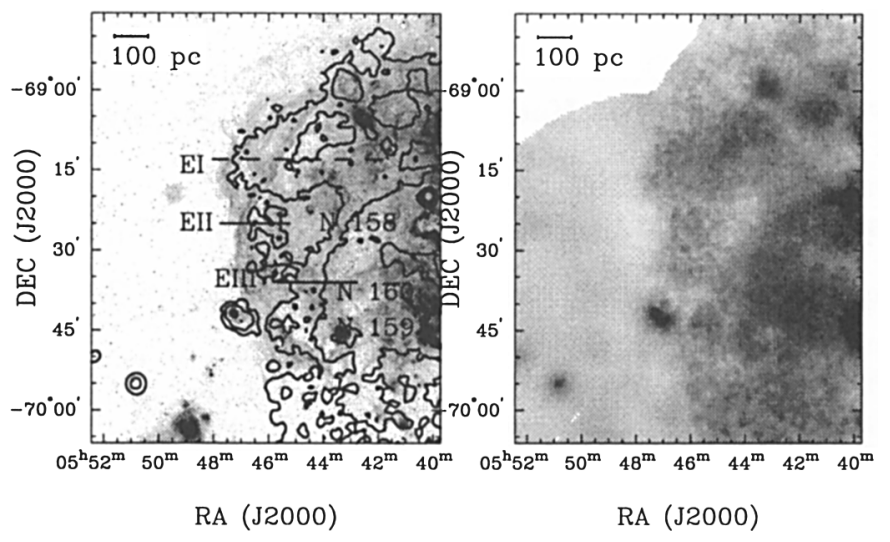

Figure 1. Left: $\mathrm{H} \alpha$ image of LMC 2 overlaid with ROSAT PSPC contours. Right: Grayscale ROSAT PSPC mosaic of LMC 2.

The ATCA aperture synthesis maps of the $\mathrm{H}$ I $21-\mathrm{cm}$ line show the neutral hydrogen toward LMC 2 to be distributed in the form of long filaments and discrete clouds. The $\mathrm{H}$ I filaments show clear correspondence with the filaments seen in the $\mathrm{H} \alpha$ image. The $\mathrm{H}$ I filaments at the periphery of LMC 2 have velocities of 250,275 , and $300 \mathrm{~km} \mathrm{~s}^{-1}$. If LMC 2 were a coherently expanding shell, we expect that the velocity extremes would be observed toward the interior of the shell, not the edge. Thus, the $\mathrm{H}$ I channel maps provide further evidence that LMC 2 is not coherently expanding.

\section{Conclusions}

We have investigated the kinematic structure of the supergiant shell LMC 2 using high-resolution echelle spectra of the $\mathrm{H} \alpha$ line and ATCA aperture synthesis maps of the $\mathrm{H}_{\mathrm{I}} 21-\mathrm{cm}$ line. High-velocity gas is detected toward X-ray bright regions. In the northern region, this high-velocity gas is indicative of localized heating by a supernova remnant. Along the western boundary, the high-velocity gas may be associated with an outflow of material from the adjacent star-forming region. However, we detect no evidence of a global expansion pattern.

\section{References}

Caulet, A., Deharveng, L., Georgelin, Y. M., \& Georgelin, Y. P. 1982, A\&A, 110,185

Goudis, C., \& Meaburn, J. 1978, A\&A, 68, 189

Meaburn, J., Marston, A. P., McGee, R. X., \& Newton, L. M. 1987, MNRAS, 225,591

Points, S. D., Chu, Y.-H., Kim, S., Smith, R. C., Snowden, S. L., Brandner, W., \& Gruendl, R. A. 1999, ApJ, 518, 298

Wang, Q. D., \& Helfand, D. J. 1991, ApJ, 379, 327 